\title{
Transient and Dynamic Stress Analysis of Functionally Graded Thick Hollow Cylinder Subjected to Thermal Shock Loading Using an Analytical Method*
}

\author{
Masoud TAHANI**, Seyed Mahmoud HOSSEINI***, Ali SAFARI** \\ and Akira TODOROKI**** \\ ** Department of Mechanical Engineering, Faculty of Engineering, Ferdowsi University of Mashhad, \\ P.O.Box 91775-1111, Mashhad, Iran \\ E-mail: mtahani@ferdowsi.um.ac.ir \\ *** Department of Industrial Engineering, Faculty of Engineering, Ferdowsi University of Mashhad, \\ P.O.Box 91775-1111, Mashhad, Iran \\ ****Department of Mechanicals Sciences and Engineering, Tokyo Institute of Technology, \\ 2-12-1 Ookayama, Meguro, Tokyo 152-8552, Japan
}

\begin{abstract}
In this paper, an analytical method is presented to investigate dynamic characteristics in a functionally graded thick hollow cylinder under thermal shock loading. Thermo-mechanical properties of functionally graded (FG) thick hollow cylinder are assumed to be temperature independent and vary continuously in the radial direction. Dynamic thermo-elastic equation of the problem is analytically solved by employing the Laplace transform and series solution. The dynamic thermo-elastic stresses in a functionally graded thick hollow cylinder subjected to axisymmetric thermal shock loading are obtained and presented in closed forms at Laplace domain. The results are obtained in time domain using the fast Laplace inverse transform method. The dynamic behaviors of thermo-elastic stresses are illustrated and discussed for various grading patterns of thermo-mechanical properties in several points across the thickness of FG cylinder and cylinder thickness parameter. The presented analytical method furnishes a ground to study the time histories of radial and hoop stresses in FG cylinders with different thickness and various exponents of volume fraction. The presented results show good agreement with published data in previous literatures.
\end{abstract}

Key words: Analytical Method, Dynamic Thermo-Elastic Stresses, Thermal Shock, Functionally Graded Materials, Time History Analysis

\section{Introduction}

One of the most important structures in various industries (such as airspace, chemical and petroleum industries) is thick hollow cylinder that is used as pressure vessels and cylindrical shells. Consequently, the dynamic characteristics of them are obviously important for engineering purposes and optimum design. In modern industries using new materials such as composites and functionally graded materials (FGMs) in cylindrical shells are increasing because of their capability to control stresses caused by thermal and mechanical loads. FGMs are new kind of composite materials whose thermo-mechanical properties vary continuously along certain directions. In the recent years, a number of 
analytical and numerical solutions have been obtained by some researchers to determine the stress distribution in cylindrical shells.

Using the Hankel asymptotic expansions, the stresses and displacements in the initial phase of applying a thermal load at the bounding surfaces of an orthotropic hollow circular cylinder are obtained by Kardomateas ${ }^{(1)}$. Wang and $\mathrm{Gong}^{(2)}$ and $\mathrm{Wang}^{(3)}$ presented an effective analytical method to analyze the histories and distributions of the dynamic thermo-elastic stresses in a homogeneous thick hollow cylinder subjected to thermal and mechanical shock loadings. Tanigawa ${ }^{(4)}$ reviewed the methods of analytical development of thermo-elastic problems for nonhomogenous materials. Cho and Kardomateas ${ }^{(5)}$ developed a closed form elasto-dynamic solution for stress field in a thick orthotropic cylindrical shell under thermal shock. They used three-dimensional linear dynamic elasticity approaches, the finite Hankel and Laplace transforms for observing the stress wave propagation phenomenon. Jabbari et al. ${ }^{(6)}$ investigated one-dimensional steady-state thermal stresses in a hollow thick cylinder made of functionally graded materials. In their analysis the material properties have been assumed as power function of radial displacement. Using the orthogonal expansion technique, thermo-elastic equations of a nonhomogenous orthotropic elastic cylindrical shell with arbitrary thickness subjected to arbitrary thermal load was solved by Ding et al. ${ }^{(7)}$. A multilayered approximation method employed to obtain the series solutions of temperature, displacement and stress fields in a functionally graded circular hollow cylinder with finite length subjected to thermo-mechanical loading by Shao ${ }^{(8)}$. Also, Shao et al. ${ }^{(9)}$ presented an analytical method based on series solution for analysis of transient thermo-mechanical stresses in a FG hollow circular cylinder with infinite length using Laplace transform. The inertia term in equation of motion was not considered by them and they considered pseudo dynamic conditions (considering elastic governing equation without inertia term in equation of motion) for their problem. Recently, they used same method for analysis of FG circular thick hollow cylinders subjected to mechanical loads and linear-type heat supply ${ }^{(10)}$. Hosseini et al. ${ }^{(11)}$ studied the vibration and dynamic analysis of functionally graded thick hollow cylinders with axisymmetry and plane strain conditions. The mean velocity of radial stress wave propagation, natural frequency and dynamic behavior of FG cylinder were presented in their work using Galerkin finite element with linear functionally graded elements and Newmark methods. To analyze the transient heat conduction in functionally graded thick hollow cylinders, an analytical method based on composition of Bessel functions was presented by Hosseini et al. ${ }^{(12)}$. Jabbari et al. ${ }^{(13)}$ analytically solved the thermo-mechanical field equations to show thermal and mechanical stress behavior in a finite length functionally graded hollow cylinder subjected to the 2-D axsymmetric steady-state thermal loads. As a numerical method Shariyat ${ }^{(14)}$ presented a Hermitian transfinite element method for nonlinear transient behavior analysis of functionally graded thick cylindrical vessels or pipes. He obtained the temperature, displacement and stress field of a FG thick cylinder with temperature-dependent material properties under thermo-mechanical loads. Also, Shariyat et al. ${ }^{(15)}$ investigated stress wave propagation characteristics in FG thick hollow cylinders with temperature-dependent properties subjected to dynamic thermo-mechanical loading. They used a second-order point-collocation method to analyze nonlinear transient thermal stresses and elastic wave propagation in FG cylinder. Also, an analytical method based on composition of Bessel's functions has been presented by Hosseini and Akhlaghi ${ }^{(16)}$ for pseudo dynamic problem in FG thick hollow cylinder subjected to transient thermal loading. Recently, Hosseini and Abolbashari ${ }^{(17)}$ presented an advanced analytical method to solve the wave motion equation in functionally graded thick hollow cylinder using composition of Bessel functions. An analytical method based on layerwise theory was presented to study displacements and stresses of functionally graded plates subjected to thermo-mechanical loadings by Tahani and Mirzababaee ${ }^{(18)}$. They solved the layerwise nonlinear governing equations analytically. 
This work presents a high strength and effective analytical method to study the thermo-elastic stress field in a functionally graded thick hollow cylinder subjected to axisymmetric thermal shock loading. The governing equations of the problem are transferred to Laplace domain and analytically solved using series solution technique. To study the dynamic behaviors of thermo-elastic stresses, the stress field responses of FG cylinder are obtained in time domain using the fast Laplace inverse transform (FLIT) method. The thermo-elastic stresses of FG cylinder are obtained in closed form at Laplace domain. The effects of various grading patterns of thermo-mechanical properties and cylinder thickness on time histories of stresses are discussed in details for several points across thickness of FG cylinder.

One of the most important differences between this paper and the other references is its capability to model any FGM cylinder subjected to arbitrary transient thermo-mechanical loading with whole governing dynamic equations (considering inertia term in equation of motion). The presented analytical method furnishes a ground to study the time histories of stresses in FG cylinders with various exponents of ceramic-metal volume fraction subjected to arbitrary transient thermo-mechanical loading. Also, the presented method has a high capability to use in wave propagation analysis of FG structures.

\section{Problem Formulation}

A functionally graded thick hollow cylinder with inner and outer radii $a$ and $b$ and infinite length is considered. The axisymmetry and plane strain conditions are assumed for problem. Consequently, strain-displacement relations can be found as follows,

$\varepsilon_{r r}(r, t)=\frac{d u(r, t)}{d r}, \varepsilon_{\theta \theta}(r, t)=\frac{u(r, t)}{r}$

where $u(r, t), \varepsilon_{r r}(r, t)$ and $\varepsilon_{\theta \theta}(r, t)$ are displacement, radial and hoop strains, respectively.

Subscripts $r r$ and $\theta \theta$ denote radial and circumferential directions. Also, the terms $t$ and $r$ show time and radius.

The dynamic thermo-elastic stresses are given by

$$
\begin{aligned}
& \sigma_{r r}(r, t)=\frac{E(r)}{(1+v)(1-2 v)}\left[(1-v) \varepsilon_{r r}(r, t)+v \varepsilon_{\theta \theta}(r, t)\right]-\frac{\alpha(r) E(r)}{(1-2 v)} T(r, t) \\
& \sigma_{\theta \theta}(r, t)=\frac{E(r)}{(1+v)(1-2 v)}\left[(1-v) \varepsilon_{\theta \theta}(r, t)+v \varepsilon_{r r}(r, t)\right]-\frac{\alpha(r) E(r)}{(1-2 v)} T(r, t)
\end{aligned}
$$

where $\sigma_{r r}(r, t), \sigma_{\theta \theta}(r, t), v, E(r), \alpha(r)$ and $T(r, t)$ are radial and hoop stresses, Poisson ratio, elastic modulus, thermal expansion coefficient and temperature of FG cylinder, respectively.

The equation of motion is

$\frac{\partial \sigma_{r r}(r, t)}{\partial r}+\frac{\sigma_{r r}(r, t)-\sigma_{\theta \theta}(r, t)}{r}=\rho(r) \frac{\partial^{2} u(r, t)}{\partial t^{2}}$

where $\rho(r)$ is the mass density.

The following mechanical boundary conditions are assumed for the problem

$$
\sigma_{r r}(a, t)=P_{a}(t)
$$

$\sigma_{r r}(b, t)=P_{b}(t)$

where $P_{a}(t)$ and $P_{b}(t)$ are hydrostatic pressures applied on the inner and outer bounding surfaces. Also, it is assumed that the temperature of the cylinder is changed as follows 
$T(r, t)=T_{0} H(t)=\left\{\begin{array}{cc}0 & t<0 \\ T_{0} & t \geq 0\end{array}\right.$

where $T_{0}$ and $H(t)$ are a constant value (reference temperature) and Heaviside step function, respectively. Furthermore, the initial conditions are considered as follows

$u(r, 0)=0,\left.\frac{\partial u(r, t)}{\partial t}\right|_{t=0}=0$

For the sake of simplicity, some dimensionless parameters can be described as

$\xi=\frac{r}{\bar{r}}, \quad \xi_{1}=\frac{a}{\bar{r}}, \quad \xi_{2}=\frac{b}{\bar{r}}, \quad \bar{E}(\xi)=\frac{E(r)}{E_{0}}, \quad \bar{\alpha}(\xi)=\frac{\alpha(r)}{\alpha_{0}}, \quad \bar{\rho}(\xi)=\frac{\rho(r)}{\rho_{0}}$

$t^{*}=\left(\frac{C_{V}}{\bar{r}}\right) t, \quad \bar{r}=\frac{a+b}{2}, \quad C_{V}=\sqrt{\frac{E}{\rho}}, \quad \bar{T}\left(\xi, t^{*}\right)=\frac{T(r, t)}{T_{0}}$

$\bar{u}\left(\xi, t^{*}\right)=\frac{u(r, t)}{\alpha_{0} T_{0} \bar{r}}, \quad \sigma_{r}\left(\xi, t^{*}\right)=\frac{\sigma_{r r}(r, t)}{\alpha_{0} T_{0} E_{0}}, \quad \sigma_{\theta}\left(\xi, t^{*}\right)=\frac{\sigma_{\theta \theta}(r, t)}{\alpha_{0} T_{0} E_{0}}$

where $C_{V}, \alpha_{0}, T_{0}, E_{0}$ and $\rho_{0}$ are reference values of stress wave propagation speed, thermal expansion coefficient, temperature, elastic modulus and density, respectively.

The equation of motion can be rewritten in dimensionless form using aforementioned non-dimensional parameters,

$$
\begin{aligned}
& \frac{\partial^{2} \bar{u}\left(\xi, t^{*}\right)}{\partial \xi^{2}}+\left(\frac{1}{\xi}+\frac{\bar{E}^{\prime}(\xi)}{\bar{E}(\xi)}\right) \frac{\partial \bar{u}\left(\xi, t^{*}\right)}{\partial \xi}+\frac{\bar{u}\left(\xi, t^{*}\right)}{\xi}\left(\frac{v}{1-v} \frac{\bar{E}^{\prime}(\xi)}{\bar{E}(\xi)}-\frac{1}{\xi}\right) \\
& -\bar{T}\left(\xi, t^{*}\right) \frac{[\bar{E}(\xi) \bar{\alpha}(\xi)]^{\prime}}{\bar{E}(\xi)}\left(\frac{1+v}{1-v}\right)-\frac{\partial \bar{T}\left(\xi, t^{*}\right)}{\partial \xi} \bar{\alpha}(\xi)\left(\frac{1+v}{1-v}\right) \\
& =\frac{(1+v)(1-2 v)}{(1-v)} \frac{\bar{\rho}(\xi)}{\bar{E}(\xi)} \frac{\partial^{2} \bar{u}\left(\xi, t^{*}\right)}{\partial t^{* 2}}
\end{aligned}
$$

Also, by using the dimensionless parameters, the boundary conditions can be expressed as

$$
\begin{aligned}
& \sigma_{r}\left(\xi_{1}, t^{*}\right)=\overline{P_{a}}\left(t^{*}\right): \\
& (1-v) \frac{\partial \bar{u}\left(\xi_{1}, t^{*}\right)}{\partial \xi}+\frac{v}{\xi_{1}} \bar{u}\left(\xi_{1}, t^{*}\right)-(1+v) \bar{\alpha}\left(\xi_{1}\right) \bar{T}\left(\xi_{1}, t^{*}\right)=\frac{(1+v)(1-2 v)}{(1-v) \bar{E}\left(\xi_{1}\right)} \overline{P_{a}}\left(t^{*}\right) \\
& \sigma_{r}\left(\xi_{2}, t^{*}\right)=\overline{P_{b}}\left(t^{*}\right): \\
& (1-v) \frac{\partial \bar{u}\left(\xi_{2}, t^{*}\right)}{\partial \xi}+\frac{v}{\xi_{2}} \bar{u}\left(\xi_{2}, t^{*}\right)-(1+v) \bar{\alpha}\left(\xi_{2}\right) \bar{T}\left(\xi_{2}, t^{*}\right)=\frac{(1+v)(1-2 v)}{(1-v) \bar{E}\left(\xi_{2}\right)} \overline{P_{b}}\left(t^{*}\right)
\end{aligned}
$$

where

$\overline{P_{a}}\left(t^{*}\right)=\frac{P_{a}\left(t^{*}\right)}{\alpha_{0} T_{0} E_{0}}$

$\overline{P_{b}}\left(t^{*}\right)=\frac{P_{b}\left(t^{*}\right)}{\alpha_{0} T_{0} E_{0}}$

and 
$\bar{T}\left(\xi, t^{*}\right)=H\left(t^{*}\right)= \begin{cases}0 & t^{*}<0 \\ 1 & t^{*} \geq 0\end{cases}$

Substituting Eq. (15) into Eqs. (10) (12), we can obtain

$$
\begin{aligned}
& \frac{\partial^{2} \bar{u}\left(\xi, t^{*}\right)}{\partial \xi^{2}}+\left(\frac{1}{\xi}+\frac{\bar{E}^{\prime}(\xi)}{\bar{E}(\xi)}\right) \frac{\partial \bar{u}\left(\xi, t^{*}\right)}{\partial \xi}+\frac{\bar{u}\left(\xi, t^{*}\right)}{\xi}\left(\frac{v}{1-v} \frac{\bar{E}^{\prime}(\xi)}{\bar{E}(\xi)}-\frac{1}{\xi}\right) \\
& -H\left(t^{*}\right) \frac{[\bar{E}(\xi) \bar{\alpha}(\xi)]^{\prime}}{\bar{E}(\xi)}\left(\frac{1+v}{1-v}\right)=\frac{(1+v)(1-2 v)}{(1-v)} \frac{\bar{\rho}(\xi)}{\bar{E}(\xi)} \frac{\partial^{2} \bar{u}\left(\xi, t^{*}\right)}{\partial t^{* 2}} \\
& (1-v) \frac{\partial \bar{u}\left(\xi_{1}, t^{*}\right)}{\partial \xi}+\frac{v}{\xi_{1}} \bar{u}\left(\xi_{1}, t^{*}\right)-(1+v) \bar{\alpha}\left(\xi_{1}\right) H\left(t^{*}\right)=\frac{(1+v)(1-2 v)}{\bar{E}\left(\xi_{1}\right)} \overline{P_{a}}\left(t^{*}\right) \\
& (1-v) \frac{\partial \bar{u}\left(\xi_{2}, t^{*}\right)}{\partial \xi}+\frac{v}{\xi_{2}} \bar{u}\left(\xi_{2}, t^{*}\right)-(1+v) \bar{\alpha}\left(\xi_{2}\right) H\left(t^{*}\right)=\frac{(1+v)(1-2 v)}{\bar{E}\left(\xi_{2}\right)} \bar{P}_{b}\left(t^{*}\right)
\end{aligned}
$$

\section{Analytical Solution}

The Laplace transform is applied to Eqs. (16) (18) with respect to time $t^{*}$, considering initial condition (8) to obtain

$$
\begin{aligned}
& \frac{\partial^{2} \tilde{u}(\xi, s)}{\partial \xi^{2}}+\left(\frac{1}{\xi}+\frac{\bar{E}^{\prime}(\xi)}{\bar{E}(\xi)}\right) \frac{\partial \tilde{u}(\xi, s)}{\partial \xi} \\
& \quad+\tilde{u}(\xi, s)\left(\frac{v}{1-v} \frac{1}{\xi} \frac{\bar{E}^{\prime}(\xi)}{\bar{E}(\xi)}-\frac{1}{\xi^{2}}-\frac{(1+v)(1-2 v)}{(1-v)} \frac{\bar{\rho}(\xi)}{\bar{E}(\xi)} s^{2}\right) \\
& \quad=\frac{[\bar{E}(\xi) \bar{\alpha}(\xi)]^{\prime}}{\bar{E}(\xi)}\left(\frac{1+v}{1-v}\right) \frac{1}{s} \\
& (1-v) \frac{\partial \widetilde{u}\left(\xi_{1}, s\right)}{\partial \xi}+\frac{v}{\xi_{1}} \widetilde{u}\left(\xi_{1}, s\right)-(1+v) \bar{\alpha}\left(\xi_{1}\right) \frac{1}{s}=\frac{(1+v)(1-2 v)}{\bar{E}\left(\xi_{1}\right)} G_{a}(s) \\
& (1-v) \frac{\partial \widetilde{u}\left(\xi_{2}, s\right)}{\partial \xi}+\frac{v}{\xi_{2}} \widetilde{u}\left(\xi_{2}, s\right)-(1+v) \bar{\alpha}\left(\xi_{2}\right) \frac{1}{s}=\frac{(1+v)(1-2 v)}{\bar{E}\left(\xi_{2}\right)} G_{b}(s)
\end{aligned}
$$

where

$\tilde{u}(\xi, s)=\mathcal{L}\left\{\bar{u}\left(\xi, t^{*}\right)\right\}$

$\frac{1}{s}=\mathcal{L}\left\{H\left(t^{*}\right)\right\}$

$$
G_{a}(s)=\mathcal{L}\left\{\overline{P_{a}}\left(t^{*}\right)\right\}
$$

$G_{b}(s)=\mathcal{L}\left\{\overline{P_{b}}\left(t^{*}\right)\right\}$

The term $\mathcal{L}$ is Laplace transform operator. According to the series method for ordinary differential equation, if the coefficient $\bar{E}^{\prime}(\xi) / \bar{E}(\xi),[\bar{E}(\xi) \bar{\alpha}(\xi)]^{\prime} / \bar{E}(\xi)$ and $\bar{\rho}(\xi) / \bar{E}(\xi)$ are analytical at $\xi=1$ and can be expressed as Taylor's series in term of $(\xi-1)$, 


$$
\begin{aligned}
& \Gamma_{1}(\xi)=\frac{\bar{E}^{\prime}(\xi)}{\bar{E}(\xi)}=\frac{1}{\bar{E}(\xi)} \frac{d \bar{E}(\xi)}{d \xi}=\sum_{n=0}^{\infty} \Gamma_{1, n}(\xi-1)^{n} \\
& \Gamma_{2}(\xi)=\frac{1}{\bar{E}(\xi)} \frac{d[\bar{E}(\xi) \bar{\alpha}(\xi)]}{d \xi}=\sum_{n=0}^{\infty} \Gamma_{2, n}(\xi-1)^{n} \\
& \Gamma_{3}(\xi)=\frac{\bar{\rho}(\xi)}{\bar{E}(\xi)}=\sum_{n=0}^{\infty} \Gamma_{3, n}(\xi-1)^{n}
\end{aligned}
$$

where

$$
\Gamma_{1, n}=\frac{1}{n !} \Gamma_{1}^{(n)}(1), \quad \Gamma_{2, n}=\frac{1}{n !} \Gamma_{2}^{(n)}(1), \quad \Gamma_{3, n}=\frac{1}{n !} \Gamma_{3}^{(n)}(1)
$$

then the solution of Eq. (19) can be expressed as Taylor's series at $\xi=1$ as following relation $^{(9)}$

$$
\widetilde{u}(\xi, s)=\sum_{n=0}^{\infty} \varphi_{n}(s)(\xi-1)^{n}
$$

Substituting Eqs.(26) and (28) into Eq.(19) and employing series properties in mathematics, one can obtain the following recurrence relation

$$
\begin{aligned}
- & (n+1)(n+2) \varphi_{n+2}=(n+1)(2 n-1) \varphi_{n+1}+\left(n^{2}-1\right) \varphi_{n} \\
& +\sum_{j=0}^{n}\left[(j-1) \varphi_{j-1}+2 j \varphi_{j}+(j+1) \varphi_{j+1}\right] \Gamma_{1, n-j}+\left(\frac{v}{1-v}\right) \sum_{j=0}^{n}\left[\varphi_{j-1}+\varphi_{j}\right] \Gamma_{1, n-j} \\
& -\left(\frac{(1+v)(1-2 v)}{1-v}\right) s^{2} \sum_{j=0}^{n}\left[\varphi_{j-2}+2 \varphi_{j-1}+\varphi_{j}\right] \Gamma_{3, n-j} \\
& -\left(\frac{1+v}{1-v}\right) \frac{1}{S}\left[\Gamma_{2, n-2}+2 \Gamma_{2, n-1}+\Gamma_{2 n}\right]
\end{aligned}
$$

From Eq.(29), all coefficients in Eq.(28) can be obtained as follows

$\varphi_{n}(s)=\psi_{1 n}(s) \varphi_{0}+\psi_{2 n}(s) \varphi_{1}+\psi_{3 n}(s)$

where $\psi_{1 n}(s), \psi_{2 n}(s)$ and $\psi_{3 n}(s)$ can be derived from the corresponding recurrence expression (29) and $\varphi_{0}$ and $\varphi_{1}$ are unknown constants, which can be determined by using the stress boundary conditions (20) and (21). So, the solution of radial displacement of the functionally graded thick hollow cylinder in Laplace domain can be expressed as

$\widetilde{u}(\xi, s)=\sum_{n=0}^{\infty}\left[\psi_{1 n}(s) \varphi_{0}+\psi_{2 n}(s) \varphi_{1}+\psi_{3 n}(s)\right](\xi-1)^{n}$

To determine the responses of stress field in time domain, the present work uses the FLIT method, which combines Fourier cosine and sine transforms ${ }^{(19)}$.

\section{Numerical Results and Discussions}

Consider an axisymmetric FG thick hollow cylinder with infinite length and inside radius $a$ and outside radius $b$. The thermo-mechanical properties of FG cylinder are graded through the radial direction. The FG cylinder is assumed to be made of a combined ceramic-metal FGM whose thermo-mechanical are varied continuously and smoothly in the radial direction from ceramic on inner surface to metal on outer surface. The material properties distribution is modeled by simple power function in term of volume fraction as follows 
$p=p_{m} V_{m}+p_{c} V_{c}=p_{m} V_{m}+p_{c}\left(1-V_{m}\right)$

where

$V_{m}=\left(\frac{r-a}{b-a}\right)^{\beta}=R^{\beta}$

and $p$ is effective material properties of FGM, $V_{m}$ and $V_{c}$ are volume fractions of metal and ceramic, and subscripts $c$ and $m$ stand for ceramic and metal, respectively. The material properties $p$ in Eq.(32) can be considered as modulus of elasticity $E$, mass density $\rho$ and coefficient of thermal expansion $\alpha$. The term $\beta$ is a non-negative volume fraction exponent that governs the distribution of the constituent materials through the thickness of FG cylinder.

Figure 1 shows the variation of volume fraction $V_{m}$ through the thickness of functionally graded cylinder for various values of the power-law index $\beta$. It can be concluded from Fig. 1 when the value of $\beta$ is zero the FG cylinder can be considered as a full metal cylinder. Also, when $\beta \rightarrow \infty$, the thermo-mechanical properties of FG cylinder converge to thermo-mechanical properties of full ceramic cylinder.

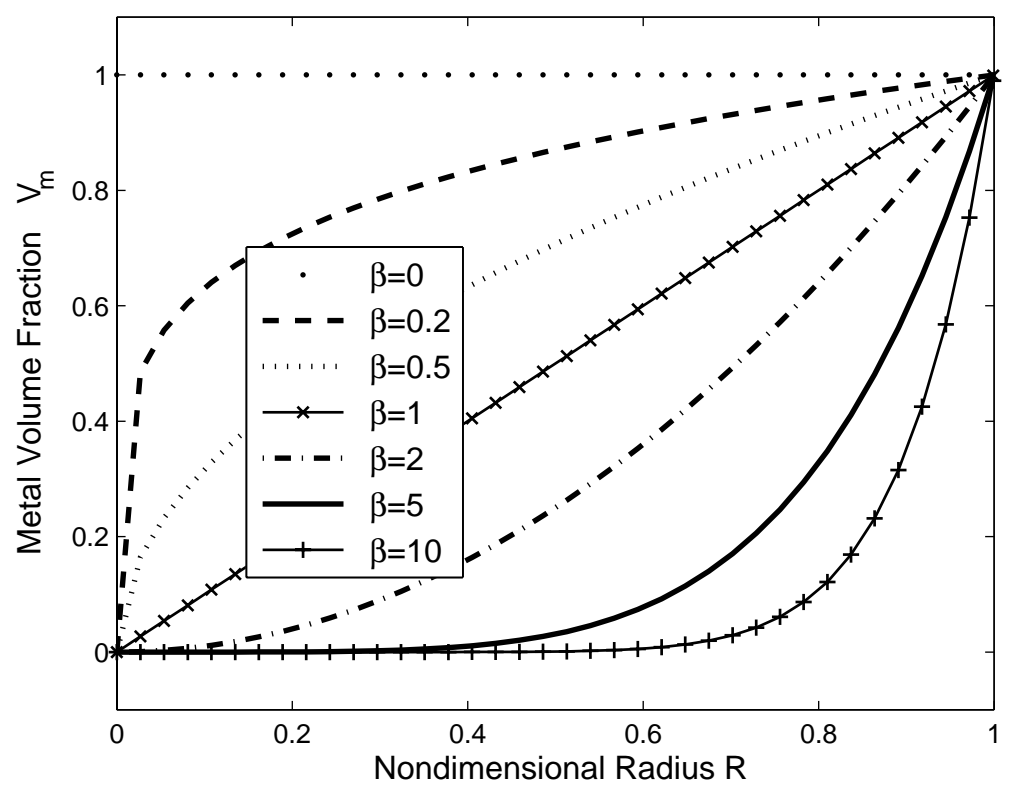

Figure 1. Effects of gradient parameter on metal volume fraction for $b / a=3$

In order to prove the validity of the presented method, the results have been compared with the analytical results obtained by $\mathrm{Wang}^{(3)}$ for isotropic thick hollow cylinders. It is important to know that the diagrams of some points on thickness of cylinder (not all of them) in [3] are digitized and drawn in Matlab software for the sake of comparison with illustrated curves based on presented analytical method.

To compare the results, the value of $\beta$ is considered to be zero and the same parameters and constants are taken into account for metal properties as;

$E=200 \mathrm{GPa}, \quad C_{V}=5000 \frac{\mathrm{m}}{\mathrm{s}}, \quad v=0.25, \quad \frac{b}{a}=2, \quad \tau=\frac{b+a}{2(b-a)} t^{*}$

Figures 2 and 3 show the comparison of the obtained results using presented analytical method and those obtained by Ref.(3). The comparison shows good agreement between obtained results from presented analytical method for FG cylinder and another analytical method for isotropic cylinder. 


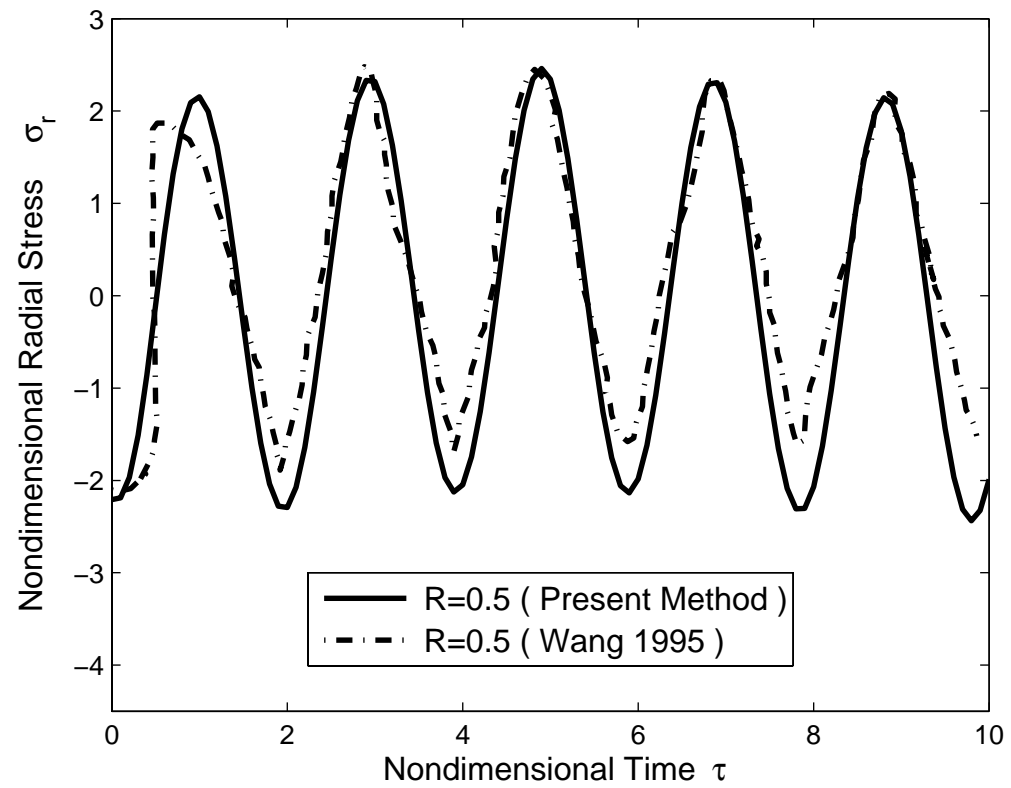

Figure 2. Time history of radial stress for a homogenous cylinder at middle point of cylinder thickness

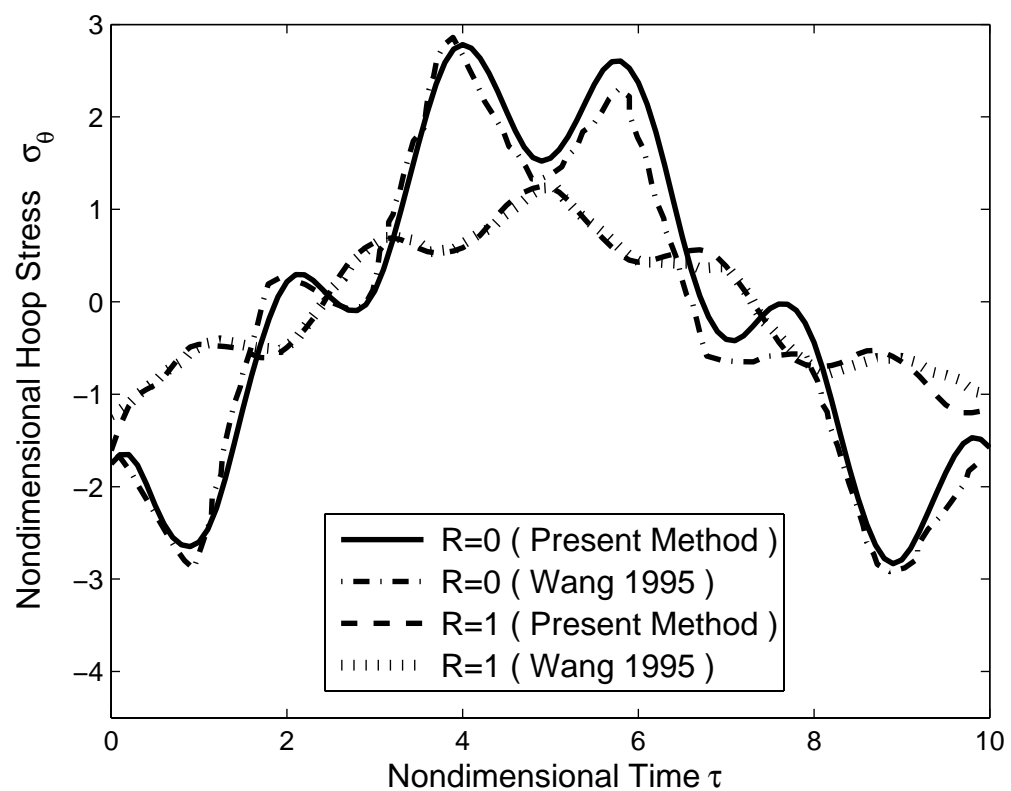

Figure 3. Time history of hoop stress for a homogenous cylinder at inner and outer surfaces

Based on results of comparison between our data (with 8 terms in series) and those published in the literatures, it can be concluded from Figs. 2 and 3 that the 8 terms of series can be used to have proper results and convergence rate.

To study the effects of thermal shock loading on dynamic behavior of FG cylinder, the bounding surfaces of FG cylinder is assumed to be traction free, thus, the corresponding mechanical boundary conditions can be expressed as

$P_{a}(t)=0, P_{b}(t)=0$

The results are obtained for a ceramic-metal functionally graded cylinder with aluminum and alumina as the metal and ceramic constituents, respectively. The material 
properties given in Table 1 are used in computing the numerical results.

Table 1. Thermo-mechanical properties of inner and outer surfaces of FG cylinder

\begin{tabular}{llll}
\hline Material & $\begin{array}{l}\text { Elasticity } \\
\text { modulus }(\mathrm{GPa})\end{array}$ & $\begin{array}{l}\text { Density } \\
\left(\mathrm{kg} / \mathrm{m}^{3}\right)\end{array}$ & $\begin{array}{l}\text { Thermal expansion } \\
\text { coefficient }\left(1 /{ }^{\circ} c\right)\end{array}$ \\
\hline Alumina (inner surface) & 380 & 3800 & $7.4 \times 10^{-6}$ \\
Aluminum(outer surface) & 70 & 2760 & $23 \times 10^{-6}$ \\
\hline
\end{tabular}

Figure 4 depicts the variation of the radial stress responses at middle point of thickness $R=0.5$ for different values of gradient parameter $\beta$. Increasing gradient parameter $\beta$ enables us to have a hollow cylinder with more ceramic volume fraction. It can be concluded from Fig.4 when the value of $\beta$ is increased, the radial stress wave propagation speed is also increased. The stress wave propagation speed $C_{V}=\sqrt{E / \rho}$ in a pure ceramic cylinder is larger than a pure metal cylinder because the elasticity modulus of the alumina is greater than the aluminum elasticity modulus. This phenomenon can be observed in Fig. 4 and the validation of presented method is further verified. Also, the amplitude of variation in Fig. 4 is directly depends on value of $\beta$. The bigger values of amplitude of variation can be seen by increasing the value of $\beta$.

Figure 5 illustrates the effect of cylinder thickness on the radial stress distribution across thickness of FG thick hollow cylinder at middle point of thickness for $\beta=2$. From Fig.5, we find that the maximum amplitude of the radial stress for cylinders with different wall thickness is almost the same. Thus, one observes that the peak values of the radial dynamic thermo-elastic stress are not related to the ratio of $b / a$. The same phenomenon can be observed in an isotropic hollow cylinder ${ }^{(3)}$.

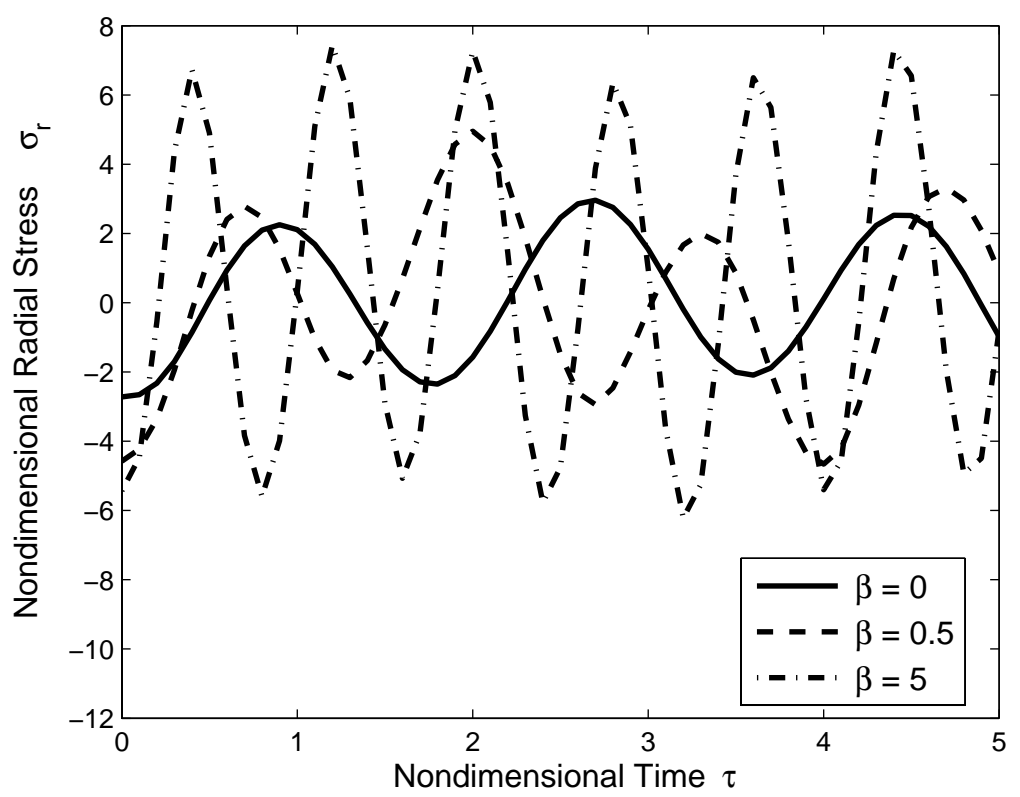

Figure 4. Time history of radial stress at middle point of thickness for $b / a=3$ and various $\beta$ 


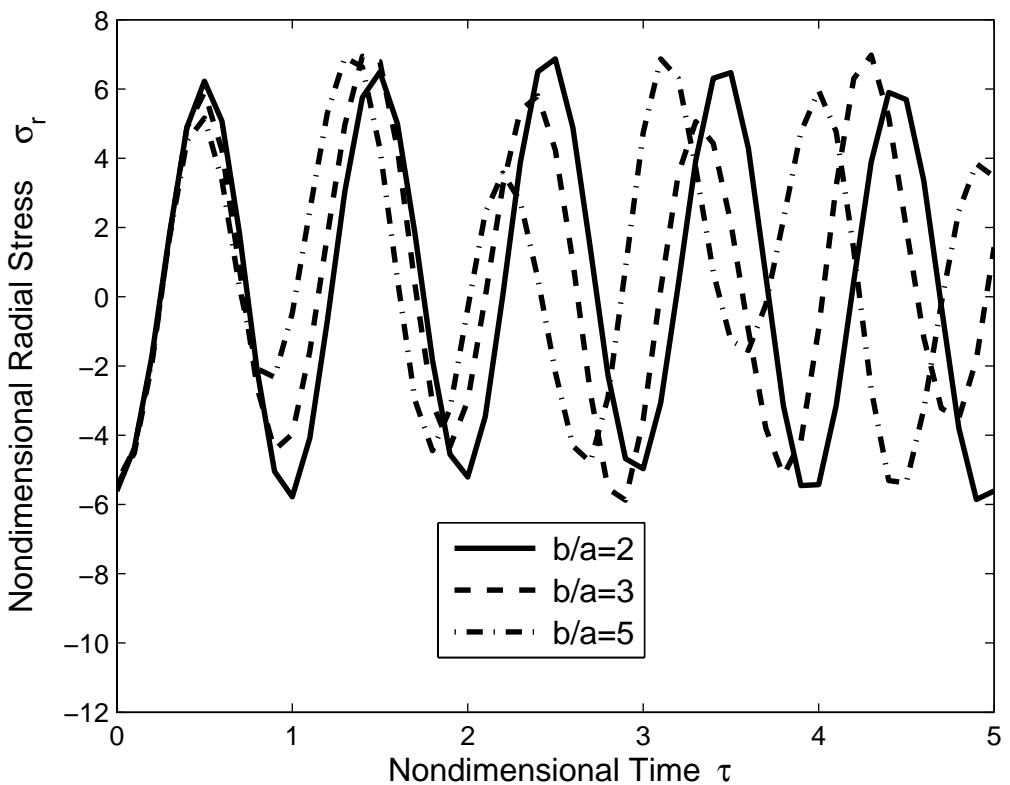

Figure 5. Time history of radial stress at middle point of thickness for $\beta=2$ and various $b / a$

Figure 6 shows the time history of hoop stress for various values of $\beta$ at inner surface of FG cylinder and $b / a=3$. Also, the similar behaviors can be found in Figs. 7 and 8 for time history of hoop stress at middle point of thickness and outer surface of FG cylinder, respectively.

From Figs.6 8, it can be concluded that the peak values of hoop stresses and amplitude of the variations directly depends on the position on thickness and value of $\beta$. Moreover, comparing these figures show that the maximum amplitudes of the hoop stress always appears at the internal surface of the FG cylinder.

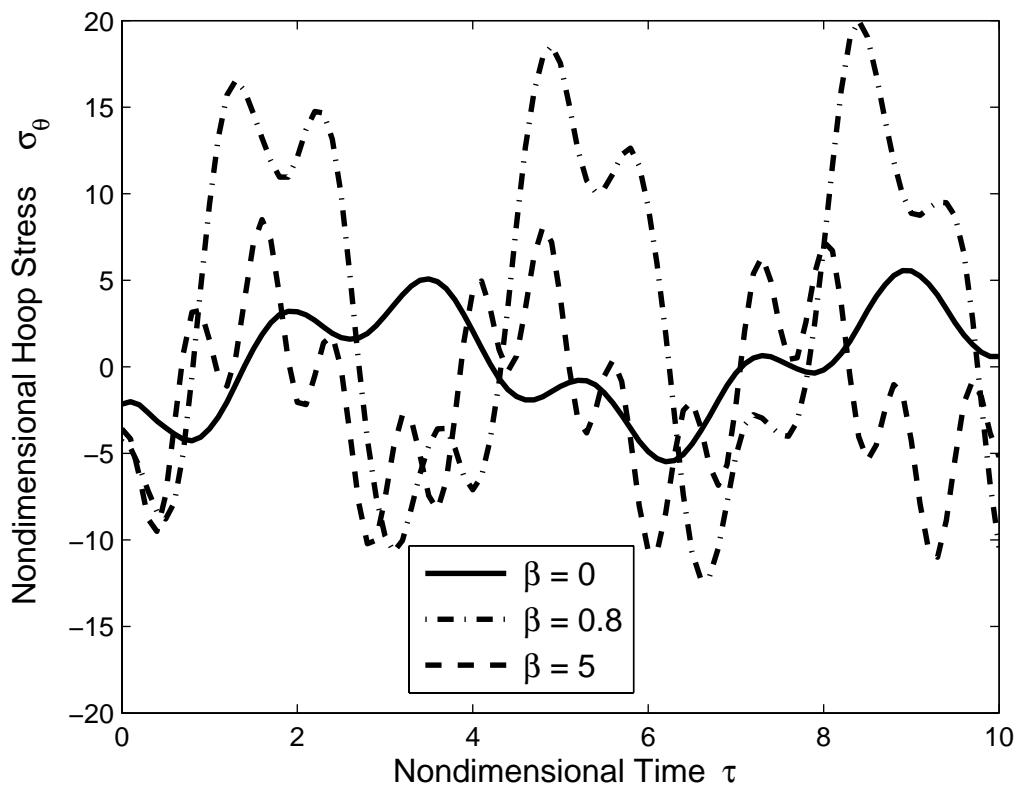

Figure 6 . Time history of hoop stress at inner surface of FG cylinder for $b / a=3$ and various $\beta$ 


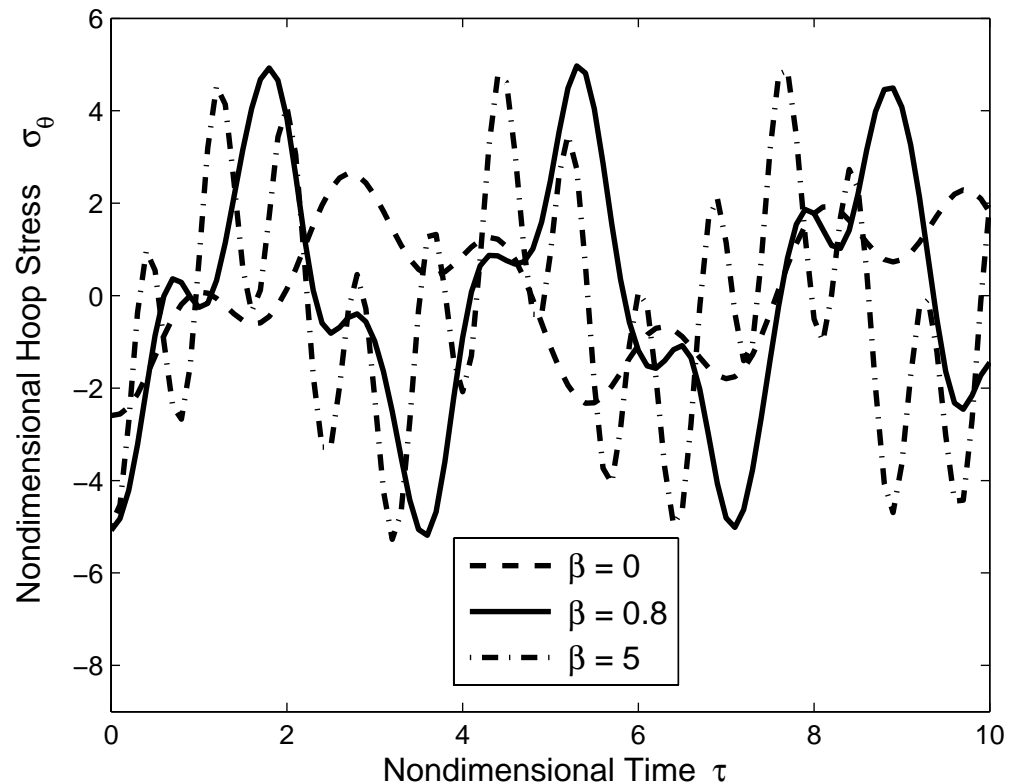

Figure 7. Time history of hoop stress at middle point of FG cylinder for $b / a=3$ and various $\beta$

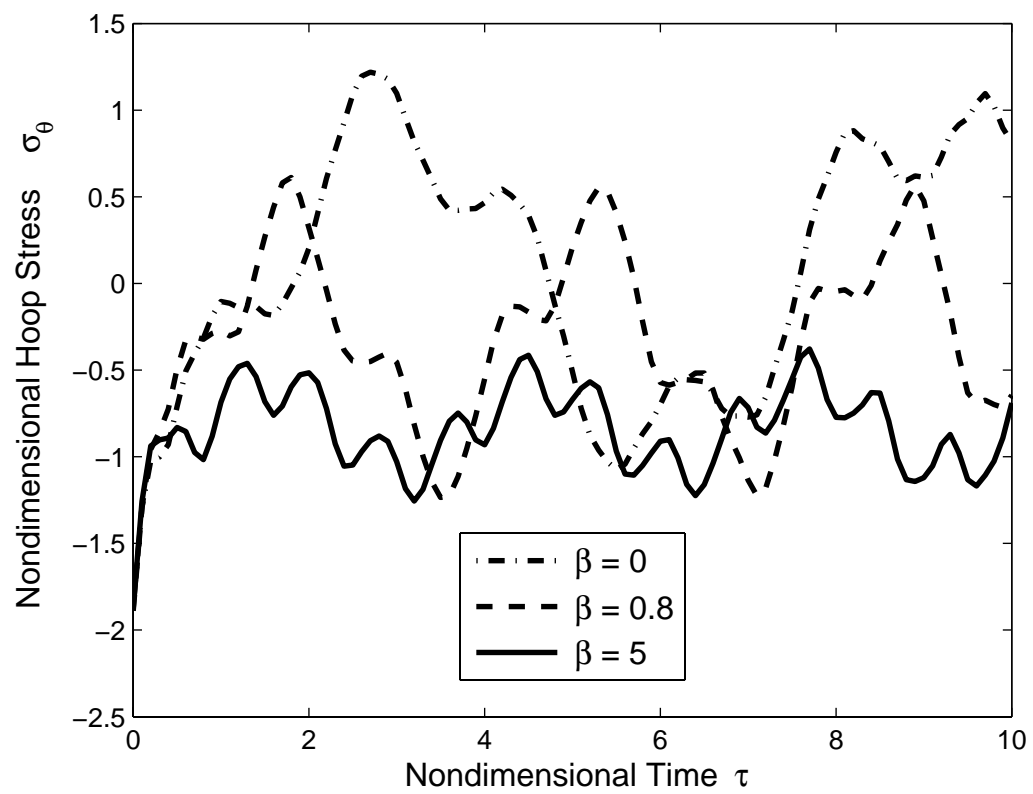

Figure 8 . Time history of hoop stress at outer surface of FG cylinder for $b / a=3$ and various $\beta$

Figure 9 shows that cylinder thickness has a significant effect on hoop stress distribution. Moreover, from Fig. 9 one observes that the maximum peak value of the hoop stress at the internal surface increases by increasing cylinder thickness. This phenomenon appears because the hoop stiffness value for a FG thick cylinder is bigger than values for a FG thin cylinder.

The effects of variation in FG cylinder thickness on time history of hoop stress at outer surface is illustrated in Fig. 10 for $\beta=2$. It can be seen in this figure that the FG cylinder thickness has not significant effect on the peak value of hoop stress at the external surface. 


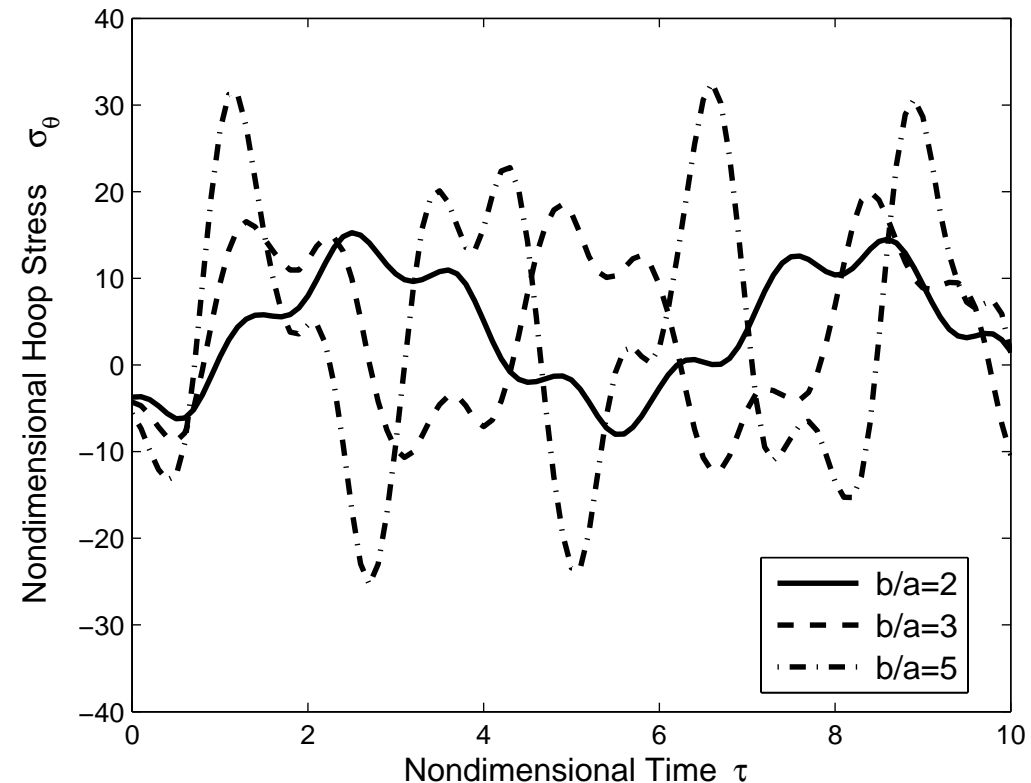

Figure 9. Time history of hoop stress at inner surface of thickness for $\beta=2$ and various $b / a$

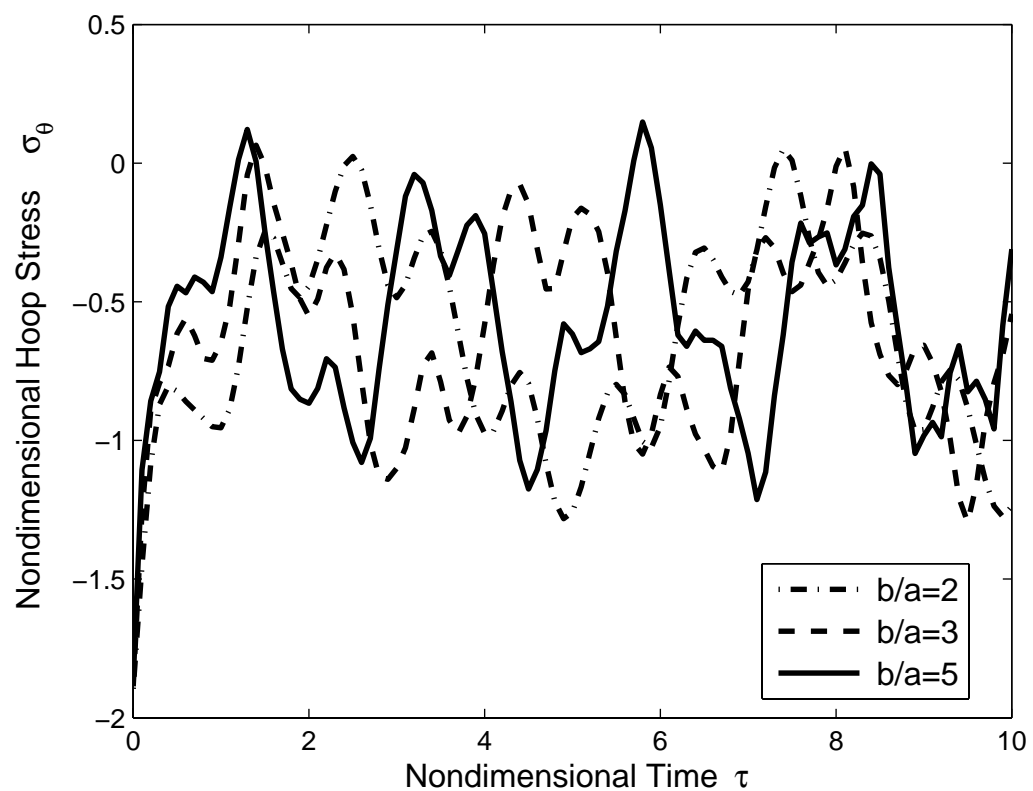

Figure 10. Time history of hoop stress at outer surface of thickness for $\beta=2$ and various $b / a$

\section{Conclusions}

In this paper, an effective general analytical solution has been presented for the dynamic analysis of thermo-elastic stresses in functionally graded thick hollow cylinder subjected to thermal shock loading. The equation of motion is analytically solved in Laplace domain using series solution and dynamic responses of FG cylinder are obtained in time domain utilizing the fast Laplace inverse transform method. The main results of this article can be outlined as follows

- The thermo-elastic displacements are obtained in closed form at Laplace domain and dynamic behavior of stress field is observed in time domain. 
- $\quad$ The time history of radial and hoop stresses are studied in details for various values of $\beta$ at several points across thickness.

- The effects of thickness and various grading patterns of thermo-mechanical properties across thickness and cylinder thickness parameter on dynamic behavior of stresses have been illustrated and discussed in details.

- The gradient parameter $\beta$ can be designed to control the amplitude of thermal stresses variation in the FG thick hollow cylinder in order to satisfy the requirement of engineering applications.

- The presented method can be used to study FG thick hollow cylinder with various grading patterns with transient boundary conditions or subjected to thermo-mechanical shock loading.

\section{References}

(1) Kardomateas, G.A., The Initial Phase of Transient Thermal Stresses Due to General Boundary Thermal Loads in Orthotropic Hollow Cylinders, Journal of Applied Mechanics, Vol.57 (1990), pp.719-724.

(2) Wang, X. and Gong, Y.N., An Elasto-Dynamic Solution for Multilayered Cylinder, International Journal of Engineering Science, Vol.30, No.1 (1992), pp.25-33.

(3) Wang, X., Thermal Shock in a Hollow Cylinder Caused by Rapid Arbitrary Heating, Journal of Sound and Vibration, (1995), pp.788-895.

(4) Tanigawa, Y., Some Basic Thermo-Elastic Problems for Nonhomogeneous Structural Materials, Journal of Applied Mechanics, Vol. 48, No.6 (1995), pp.287-300.

(5) Cho, H. and Kardomateas, G.A., Thermal Shock Stress Due to Heat Convection at the Bounding Surface in a Thick Orthotropic Cylindrical Shell, International Journal of Solid and Structures, Vol.38(2001), pp.2769-2788.

(6) Jabbari, M., Sohrabpour, S. and Eslami, M.R., Mechanical and Thermal Stresses in a Functionally Graded Hollow Cylinder Due to Radially Symmetric Loads, International Journal of Pressure Vessels and Piping, Vol.79(2002), pp.493-497.

(7) Ding, H.J., Wang, H.M. and Chen, W.Q., A Solution of a Nonhomogeneous Orthotropic Cylindrical Shell for Axisymmetric Plane Strain Dynamic Thermo-Elastic Problems, Journal of Sound and Vibration, Vol.263 (2003), pp.815-829.

(8) Shao, Z.S., Mechanical and Thermal Stresses of a Functionally Graded Circular Hollow Cylinder with Finite Length, International Journal of Pressure Vessels and Piping, Vol.82 (2005), pp.155-163.

(9) Shao, Z.S., Wang, T.J. and Ang, K., Transient Thermo-Mechanical Analysis of Functionally Graded Hollow Circular Cylinders, Journal of Thermal Stresses, Vol.30, No.1 (2007), pp.81-104.

(10) Shao, Z.S. and Ma, G.W., Thermo-Mechanical Stresses in Functionally Graded Circular Hollow Cylinder with Linearly Increasing Boundary Temperature, Composite Structures, Vol.83 (2008), pp.259-65.

(11) Hosseini, S.M., Akhlaghi, M. and Shakeri, M., Dynamic Response and Radial Wave Propagation Velocity in Thick Hollow Cylinder Made of Functionally Graded Materials, Engineering Computations, Vol.24,No.3(2007), pp.288-303.

(12) Hosseini, S.M., Akhlaghi, M. and Shakeri, M., Transient Heat Conduction in Functionally Graded Thick Hollow Cylinders by Analytical Method, Heat Mass Transfer, Vol.43 (2007), pp.669-675.

(13) Jabbari, M., Bahtui, A. and Eslami, M.R., Axisymmetric Mechanical and Thermal Stress in Thick Short Length FGM Cylinders, International Journal of Pressure Vessels and Piping, Vol.86(2009), pp.296-306.

(14) Shariyat, M., A nonlinear Hermitian Transfinite Element Method for Transient 
Behavior Analysis of Hollow Functionally Graded Cylinders with Temperature-Dependent Materials under Thermo-Mechanical Loads, International Journal of Pressure Vessels and Piping, Vol.86 (2009), pp.280-289.

(15) Shariyat, M., Lavasani, S.M.H. and Khaghani, M., Nonlinear Transient Thermal Stress and Elastic Wave Propagation Analyses of Thick Temperature-Dependent FGM Cylinders, Using a Second-Order Point-Collocation Method, Applied Mathematical Modelling, (2009), doi:10.1016/j.apm.2009.07.007.

(16) Hosseini, S.M. and Akhlaghi, M., Analytical Solution in Transient Thermo-Elasticity of Functionally Graded Thick Hollow Cylinders (Pseudo-Dynamic Analysis), Mathematical Method in the Applied Sciences, 2009, DOI: 10.1002/mma.

(17) Hosseini, S.M. and Abolbashari, M.H., General Analytical Solution for Elastic Radial Wave Propagation and Dynamic Analysis of Functionally Graded Thick Hollow Cylinders Subjected to Impact Loading, Acta Mechanica., 2009, DOI 10.1007/s00707-009-0237-5.

(18) Tahani, M. and Mirzababaee, S.M., Nonlinear Analysis of Functionally Graded Plates in Cylindrical Bending Under Thermomechanical Loadings Based on a Layerwise Theory, European Journal of Mechanics A/Solids, Vol. 28 (2009), pp.248-256.

(19) Durbin F., Numerical Inversion of Laplace Transforms: An Efficient Improvement to Dubner and Abate's Method, Computer Journal, Vol.17 (1974), pp.371-376. 